\title{
Global software engineering and the Brazilian perspective
}

\author{
Rafael Prikladnicki • Erran Carmel • \\ Jorge Luis Nicolas Audy
}

Published online: 3 June 2010

(C) The Brazilian Computer Society 2010

Since the rise of the Internet in the 1990s, we have entered the era of Global Software Engineering (GSE). High-tech companies are now located in nearly 100 nations. Many Latin America nations have flourished in this new era: Costa Rica lured Intel, Argentina lured EDS (now HP), and Brazil has lured many global high-tech firms and many investments in the IT sector. The rules developed by the Brazilian government in order to reduce tariff barriers, exclude the market reserve and reestablish the National Computer Policy have actively added to the position of Brazil as an interesting option to receive foreign investments in the high technology sector.

The government's goal of supporting industries already based in Brazil and the need to create an attractive environment for the foreign capital led to the creation of the Computer Tax Incentive Law (Laws 8248/91, 10176/01, and 11077/04). This law allows companies to invest in research and development projects (previously approved) rather than pay these funds as income taxes. This measure strongly bolstered the creation of new software companies and also attracted new companies to Brazil that produce computer goods in several regions of the nation today. Among those companies are several software development centers of large foreign companies, which saw a way of reducing costs

\footnotetext{
R. Prikladnicki ( $₫) \cdot$ J.L.N. Audy

Computer Science School, PUCRS, Porto Alegre, Brazil

e-mail: rafaelp@pucrs.br

J.L.N. Audy

e-mail: audy@pucrs.br

E. Carmel

Kogod School of Business, American University,

Washington, DC, USA

e-mail: carmel@american.edu
}

through total or partly distribution of their software development areas by taking advantage of incentive offered by the government and technical preparation of Brazilian computer professionals.

The Internet has facilitated a massive dispersion of hightech work. Seeking lower costs and access to skilled resources, many organizations have remotely located software development facilities. As a result, software development is increasingly a multi-site, globally-distributed undertaking. However, much coordination is still needed across distance. Problems and challenges intrinsic to software development processes become more critical in GSE. The diversity of culture and the dispersion over time and space require novel techniques, tools, and practices from many disciplines to overcome challenges and to use the advantages and opportunities that GSE offers.

In this special section, we gathered three papers that present innovative ideas on how to cope with the growing complexity of effectively managing GSE projects. We received papers from around the world. Each paper was reviewed by at least three expert referees. The guest editors would like to thank all the reviewers and authors of submitted papers to this special section. The three papers of this Special Section are related to important challenges faced by distributed project team members: task allocation, awareness, and project management. The papers are described briefly here.

The first paper is "A Multi-Criteria Distribution Model for Global Software Development Projects”, written by Ansgar Lamersdorf and Jürgen Münch, from the University of Kaiserslautern, presents a customizable multi-criteria model for task allocation in global software development/GSE projects. Based on an analysis of the state of the practice, 
a set of requirements was derived and used for evaluating existing task allocation models from different domains. Experience from applying the model is also presented.

The second paper is entitled "Improving Design Evolution Awareness in Software Development", written by Rafael Cepeda, Andréa Magdaleno, Leonardo Murta, and Cláudia Werner, all from the Federal University of Rio de Janeiro (COPPE/UFRJ). The paper presents an approach based on software visualization that can detect and externalize design evolution made in a software project during its initial development or at any further phase. The approach was evaluated with free/open source software (FOSS) projects.
The third paper is authored by Valentine Casey, from Bournemouth University, and is entitled "Virtual Software Team Project Managament". Based on extensive research conducted by the author, the purpose of the study was to identify key factors and variables which were directly relevant to the effective operation and management of virtual software teams. The author presents six specific project management related areas that need to be addressed to facilitate successful virtual team operation: Organizational Virtual Team Strategy, Risk Management, Infrastructure, Implementation of a Virtual Team Process, Team Structure, and Organization and Conflict Management. 